\title{
Bose-Fermi mixtures of self-assembled filaments of fermionic polar molecules
}

\author{
M. Klawunn ${ }^{1}$, J. Duhme ${ }^{1}$ and L. Santos ${ }^{1}$ \\ ${ }^{1}$ Institut für Theoretische Physik, Leibniz Universität Hannover, Appelstr. 2, D-30167, Hannover, Germany
}

\begin{abstract}
Fermionic polar molecules in deep 1D optical lattices may form self-assembled filaments when the electric dipoles are oriented along the lattice axis. These composites are bosons or fermions depending on the number of molecules per chain, leading to a peculiar and complex Bose-Fermi mixture, which we discuss in detail for the simplest case of a three-well potential. We show that the interplay between filament binding energy, transverse filament modes, and trimer Fermi energy leads to a rich variety of possible scenarios ranging from a degenerate Fermi gas of trimers to a binary mixture of two different types of bosonic dimers. We study the intriguing zero temperature and finite temperature physics of these composites for the particular case of an ideal filament gas loaded in $1 \mathrm{D}$ sites, and discuss possible methods to probe these chain mixtures.
\end{abstract}

PACS numbers: 03.75.Lm,05.30.Jp

A new generation of experiments is starting to explore systems where the dipole-dipole interaction (DDI) plays a significant and possibly dominant role. Due to the longrange anisotropic character of the DDI, dipolar quantum gases offer a fascinating novel physics [1, 2]. Exciting phenomena have been recently reported for experiments on Bose-Einstein condensates (BECs) of atomic magnetic dipoles, especially on Chromium BEC 3], but also on spinor Rubidium BECs [4], Potassium [5] and Lithium [6]. Magnetic atomic dipoles are however rather weak. On the contrary heteronuclear molecules, especially at their lowest rovibrational state, may present a very large electric dipole moment ( $\gtrsim 1$ Debye) [7, [8,, 9$]$. Although quantum degeneracy has not been yet achieved, the rapid pace of development allows to expect degenerate gases of polar molecules in the next future. These gases are expected to be largely dominated by the DDI.

Deep 1D optical lattices (formed by counterpropagating lasers) may slice a gas into non-overlapping samples. For non-dipolar (short-range interacting) particles these non-overlapping gases may be considered as independent parallel experiments. The situation is completely different in dipolar gases, since the DDI leads to inter-site interactions. For weak DDI (e.g. atomic magnetic dipoles) these inter-site interactions lead to scattering between BECs at different sites [10], collective excitations shared by non-overlapping sites [11], or damping of Bloch oscillations [5]. For bosonic polar molecules the non-local dipolar effects may be much stronger, leading to fascinating effects as pair-superfluidity for ladder-like lattices [12] and filament BEC [13].

Filamentation is indeed an interesting possibility introduced by the DDI. This phenomenon, first suggested in the context of ferrofluids by de Gennes and Pincus 14, has attracted a considerable theoretical interest for the case of classical dipoles [15]. Dipolar chains in classical ferrofluids were recently observed in superparamagnetic iron colloids 16 and single-domain magnetite colloids [17]. In Ref. [13] it was shown that a similar filamentation process may occur for bosonic polar molecules

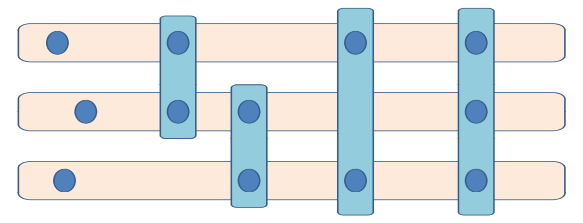

Figure 1: Polar fermionic molecules in a three-well potential may remain unpaired, form fermionic trimers, or bosonic dimers between nearest neighbors or next-nearest neighbors.

in deep lattices, which may organize into chains sustained by an attractive inter-site DDI, forming a dipolar chains liquid (DCL) which may Bose-condense [13].

In this Letter we consider DCLs of fermionic polar molecules. Far from being a trivial extension of the bosonic case, fermionic polar molecules lead to a very different and rich physics. Whereas for bosonic molecules the chains are obviously bosons, for fermionic molecules the bosonic or fermionic character of the filaments depends on the number of molecules in the chain. This has particularly relevant consequences when the number of available lattice sites is odd. Here we focus on the simplest non-trivial case, namely a three-well potential (Fig. 1). For simplicity we restrict our discussion to the ideal gas regime, where inter-filament interactions are neglected. Although this approximation is of limited quantitative validity (and would demand mesoscopic samples in specific 1D arrangements as discussed below), it contains already many of the qualitatively new features which may be expected for more general scenarios of polar Fermi molecules in deep 1D and 2D optical lattices. In particular, the competition between trimer/dimer binding and trimer Fermi energy results in a non-trivial dependence of the character of the DCL as a function of the number of molecules per site $N$. If $N$ is smaller than a critical $N_{c}$ the DCL is a Fermi-degenerate gas of trimers. However for $N>N_{c}$ the trimers coexists with a Bose mixture formed by pseudo-spin- $1 / 2$ dimers and spinless dimers, leading to a peculiar Bose-Fermi mixture. We 
show that these Bose-Fermi DCLs may be probed by monitoring the spatial distribution of the molecules.

We consider fermionic polar molecules with mass $m$ and electric dipole $d$ in a deep three-well potential along the $z$-direction, with inter-site spacing $\Delta$. This arrangement may be created by e.g. selectively emptying all sites of a strong 1D optical lattice except three neighboring ones. The potential barriers are large-enough to prevent any inter-site hopping. Due to reasons discussed below, the analysis of the problem simplifies notably if the gas is considered as strongly confined along the $y$-direction (e.g. in a single node of a lattice as that in the $z$-direction). Along the remaining $x$-direction we consider a shallow harmonic confinement with frequency $\omega$. The molecules interact via the DDI $V_{d}(\mathbf{r})=d^{2}\left(1-3 \cos ^{2} \theta\right) / r^{3}$, where $\theta$ is the angle formed by $\mathbf{r}$ with the dipole orientation. We assume that the dipoles form an angle $\alpha$ with the $z$ direction, such that $\sin ^{2} \alpha=1 / 3$. Although this particular orientation and the $1 \mathrm{D}$ character of the sites are not needed for the formation of the DCL gas, which may occur also in stacks of 2D sites [13], this particular scenario allows both for a strong attraction between dipoles placed on top of each other and for a vanishing DDI between molecules at the same site. This largely reduces the inter-filament interaction, allowing for a simplified ideal gas scenario, as discussed below.

The attraction between polar molecules placed on top of each other may be strong-enough to bind two or more polar molecules into self-assembled chains (Fig. 1). Whereas for bosonic molecules these chains are in any case bosons [13], for fermionic molecules the fermionic/bosonic character of the filaments depends on the odd/even number of molecules in a given chain. In particular, the three-well configuration allows for fermionic trimers (and of course monomers), and two different kinds of bosonic dimers, namely those between two molecules at nearest neighbors (type I dimers), and those between two molecules at the uppest and lowest site (type II dimers) (Fig. 11). Note that dimers I are actually pseudo-spin- $1 / 2$ bosons, since dimers in sites 1 and 2 are not equivalent to dimers in sites 2 and 3 .

The ground-state of a single filament of $M$ molecules is calculated as for bosonic molecules [13], and hence we just sketch for completeness the basic ideas. Let $\mathbf{r}_{j}\left(\hat{\mathbf{p}}_{j}\right)$ be the position (momentum) operator of a molecule at site $j$. Introducing $\hat{\mathbf{P}}=\sum_{j=1}^{M} \hat{\mathbf{p}}_{j}, \mathbf{R}=\sum_{j=1}^{M} \mathbf{r}_{j} / M$, $\mathbf{q}_{j}=\mathbf{p}_{j}-\mathbf{P}, \mathbf{s}_{j}=\mathbf{r}_{j}-\mathbf{R}=\left\{x_{j}, y_{j}, z_{j}\right\}$, the Hamiltonian splits into $\hat{H}=\hat{H}_{C M}+\hat{H}_{r e l}$, where $\hat{H}_{C M}=\hat{\mathbf{P}}^{2} / 2 M m+$ $M m \omega^{2} R_{x}^{2} / 2$ describes the filament center-of-mass and

$$
\begin{aligned}
\hat{H}_{r e l} & =\sum_{j=1}^{M}\left[\frac{\hat{\mathbf{q}}_{j}^{2}}{2 m}+\frac{m}{2}\left(\omega_{\perp}^{2}\left(y^{2}+z_{j}^{2}\right)+\omega^{2} x^{2}\right)\right] \\
& +\sum_{i, j>i} V_{d}\left[\mathbf{s}_{i}-\mathbf{s}_{j}\right] .
\end{aligned}
$$

the relative motion. The on-site $y z$ confinement is approximated by a strong isotropic harmonic oscillator of frequency $\omega_{\perp}$. The wavefunction of the $j$-th molecule is chosen as $\psi_{j}\left(x_{j}-x_{j 0}\right) \varphi_{j}\left(y_{j}\right) \varphi_{j}\left(z_{j}-z_{j 0}\right)$, where $\varphi_{j}(\eta)=\exp \left(-\eta^{2} / 2 l_{\perp}^{2}\right) / \sqrt{l_{\perp} \sqrt{\pi}}$, and $\psi_{j}(\eta)=$ $\exp \left(-\eta^{2} / 2 R_{0}^{2}\right) / \sqrt{\sqrt{\pi} R_{0}}$, with $l_{\perp}=\sqrt{\hbar / m \omega_{\perp}}$ and $R_{0}$ is the variational width of the $x$ wavepackets. For deep lattices one may approximate $l_{\perp} \rightarrow 0$ (energy corrections are $\lesssim 1 \%$ for depths $>14$ recoil energies $\left.\hbar^{2} \pi^{2} / 2 m \Delta^{2}\right)$.

Minimizing the energy of straight filaments $\left(x_{j 0}=0\right)$ with respect to $R_{0}$ we obtain the filament binding energy [18. We denote as $-E_{T},-E_{D, I}$ and $-E_{D, I I}$ the binding energies for, respectively, trimers, dimers I, and dimers II. These energies grow with the dipole strength $U_{0}=$ $m d^{2} / \hbar^{2} \Delta$. There exists a critical $U_{0}^{*}$ such that for $U_{0}<$ $U_{0}^{*}$ the composites unbind $\left(R_{0} \gtrsim l_{H O}=\sqrt{\hbar / m \omega}\right)$. Note that $U_{0}^{*}(T)<U_{0}^{*}(D, I) \ll U_{0}^{*}(D, I I)$ (Fig. (2)) due to the different strength of the DDI in each composite. In the following we consider the regime $U_{0}>U_{0}^{*}(D, I I)$, where $R_{0} \ll l_{H O}$ for all of the possible composites of Fig. 1.

Transverse filament excitations contribute to the gas entropy, being relevant at finite temperature $T$. In addition, and contrary to the case of bosonic molecules [13], transverse modes are important for fermionic molecules also at very low $T$ since they may significantly reduce the trimer Fermi energy. For a chain of $M$ molecules, we obtain the low-lying modes $\xi_{\nu=1, \ldots, M}$ after expanding the chain energy $E$ around its minimum, and diagonalizing $\partial^{2} E / \partial x_{j} \partial x_{j^{\prime}}$, where $j, j^{\prime}=1, \ldots, M$.

In the following we consider the filament statistics, assuming an ideal filament gas. This largely simplifies the analysis of the problem, while allowing for the discussion of key qualitative features of these systems, in particular the competition between different Bose and Fermi composites. This approximation is just quantitatively valid for mesoscopic samples in the arrangement discussed above, as we discuss at the end of this Letter.

The fermionic or bosonic character of the chains is reflected by the average occupations for trimers, dimers I, dimers II and monomers:

$$
\begin{aligned}
N_{T}\left(n, \nu_{T}\right) & =\left[e^{\beta\left[-E_{T}+\xi_{\nu_{T}}+\epsilon_{n}-\left(2 \mu_{1}+\mu_{2}\right)\right]}+1\right]^{-1}(2) \\
N_{D, I}\left(n, \nu_{D, I}\right) & =\left[e^{\beta\left[-E_{D, I}+\xi_{\nu_{D}, I}+\epsilon_{n}-\left(\mu_{1}+\mu_{2}\right)\right]}-1\right]^{-1}(3) \\
N_{D, I I}\left(n, \nu_{D, I I}\right) & =\left[e^{\beta\left[-E_{D, I I}+\xi_{\nu_{D}, I}+\epsilon_{n}-2 \mu_{1}\right]}-1\right]^{-1}(4) \\
N_{S, j}(n) & =\left[e^{\beta\left[\epsilon_{n}-\mu_{j}\right]}+1\right]^{-1}
\end{aligned}
$$

where $N_{S, j}$ denotes the average occupation of individual molecules at site $j, \xi_{\nu_{T ; D, I ; D, I I}}$ the transverse filaments modes of the different composites, $\epsilon_{n}=\hbar \omega(n+1 / 2)$ the harmonic oscillator levels and $\beta=1 / k_{B} T$ the inverse temperature. In the previous expressions we have assumed symmetric configurations such that the number of dimers I in sites 1-2 is the same as the number of dimers I in sites $2-3$, and equal to $N_{D, I}\left(n, \nu_{D, I}\right)$. Note that 


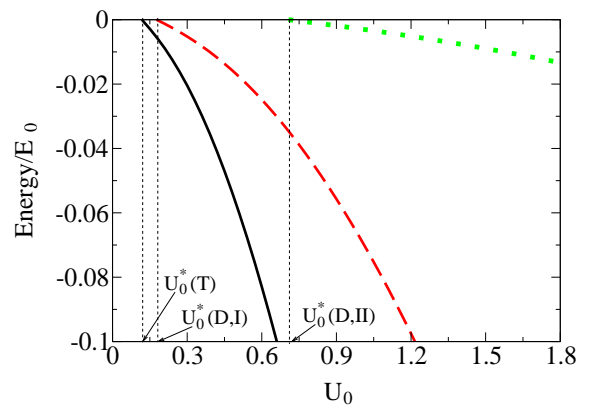

Figure 2: Binding energy (in units of $E_{0}=\hbar^{2} / m \Delta^{2}$ ) of the different composites of Fig. 11 as a function of $U_{0}=m d^{2} / \hbar^{2} \Delta$.

$\mu_{1}=\mu_{3}$ is the chemical potential for molecules at the uppest and lowest sites, whereas $\mu_{2}$ denotes the chemical potential for molecules in the middle site. These different chemical potentials are necessary to fulfill the normalization conditions, in which we assume $N$ molecules per lattice site. Imposing symmetry between the uppest and the lowest sites, these conditions acquire the form:

$$
\begin{aligned}
& N=N_{T}+N_{D, I}+N_{D, I I}+N_{S, 1}, \\
& N=N_{T}+2 N_{D, I}+N_{S, 2},
\end{aligned}
$$

where $N_{T}, N_{D, I}, N_{D, I I}, N_{S, 1}$ and $N_{S, 2}$ denote respectively the total number of trios, dimers I in sites 1-2 (or 2-3), dimers II, monomers in site 1 (or 3 ) and monomers in site 2. From (6) and (7) we obtain $\mu_{1}(N, T)$ and $\mu_{2}(N, T)$, and from (2) 5) the occupation numbers.

Due to the attractive DDI between molecules in the filament, the most bound chain is the trimer. The difference in binding between dimers and trimers induces that for sufficiently small $N$ and at low-enough T the DCL becomes a degenerate Fermi gas of trimers. The trimers fill up oscillator levels (and also transverse trimer modes) up to the corresponding Fermi energy $E_{F}(N)$, which equals $N \hbar \omega$ for rigid filaments but it is actually smaller due to the transverse trimer modes. However, if the number of molecules per site is sufficiently large, the growth in Fermi energy overcomes the binding energy difference. This transition may be easily estimated by comparing the average energy per molecule for the case of two trimers and that for the case of 2 dimers I and one dimer II. This leads to a condition for the critical number of molecules per site $N_{c}\left(U_{0}, \omega\right), E_{F}\left(N_{c}\right)=2 E_{T}-3\left(E_{D, I}+E_{D, I I}\right) / 2$ (which we have confirmed numerically). Note that $N_{c}$ grows with growing $U_{0}$ and decreasing $\omega$. For $N<N_{c}$ the DCL is a degenerate trimer gas, whereas for $N>N_{c}$ the trimer gas coexists with a mixture of pseudo-spin- $1 / 2$ bosons (dimers I) and spin-less bosons (dimers II).

The peculiar properties of the DCL translate into the spatial molecular distribution integrated over the three sites. For $N<N_{c}$ and $N<\xi_{1_{T}} / \omega$, only trimers in their internal ground state are formed, and hence the gas behaves as a spin-less Fermi gas of particles of mass $3 m$, presenting a Thomas-Fermi density profile $\left(1-(x / R)^{2}\right)^{1 / 2}$
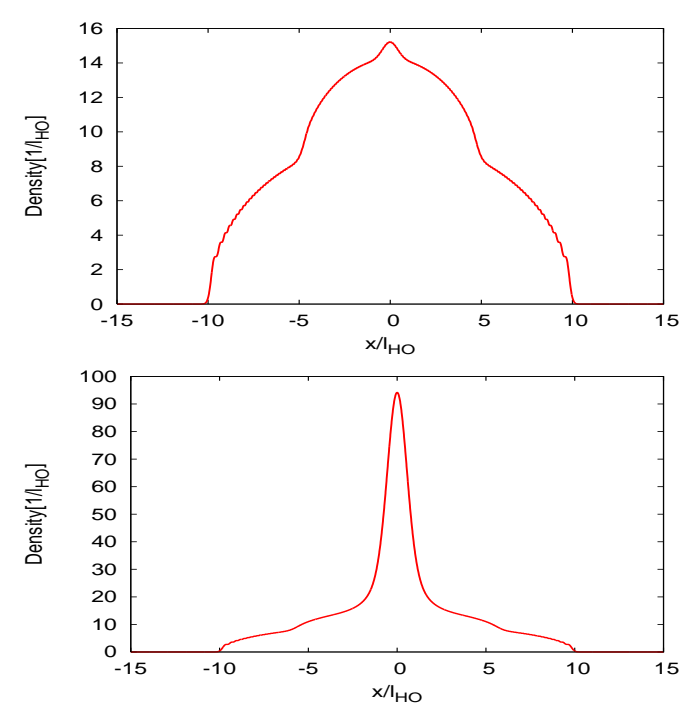

Figure 3: Integrated density profiles of the molecules, for $\xi_{1_{T}} / \omega<N<N_{c}$ (top), and $N>N_{c}$ (bottom). We consider $U_{0}=2, \omega / 2 \pi=1 \mathrm{~Hz}, m=100 \mathrm{amu}$, which lead to $N_{c}=230$.

with $R / l_{H O}=\sqrt{2 N / 3}$. For $\xi_{1_{T}} / \omega<N<N_{c}$, the DCL is still a trimer gas, but transversal modes may be populated. In that case the density profile departs from the Thomas-Fermi profile (Fig. 3 top), due to the appearance of internally excited trimers in low harmonic oscillator levels. For $N>N_{c}$ the density profile changes dramatically. Note that since we consider 1D gases, dimer BEC is strictly speaking precluded. However, due to finite size the dimers quasi-condense (at low-enough $T$ ) occupying the few lowest levels of the harmonic oscillator. Hence when $N$ surpasses $N_{c}$ a Bose cloud nucleates at the trap center. As a result the distribution of the polar molecules shows a Gaussian-like peak at the trap center (Fig. 3, bottom).

The DCL presents as well an intriguing finite temperature physics due to the role of filament modes and the different binding energy of dimers and trimers. This is particularly clear from a finite $T$ analysis of a DCL with $N<N_{c}$ (Fig. (4). Note that whereas at very low $T$ the DCL is purely a trimer Fermi gas, at finite $T$ it becomes more favorable to populate dimers than to populate higher excited trimer states. As a consequence the system presents a striking thermal enhancement of the bosonic modes. Interestingly, contrary to the standard situation, this leads to a maximal central peak density for a given finite $T$. For even larger $T$ the central density decreases again due to the occupation of dimers at higher oscillator modes, and the breaking of the filaments into individual molecules.

For $N \gg N_{c}$ and $U_{0}>U_{0}^{*}(D, I I)$ the DCL is at low T a basically pure Bose gas of dimers I and II (except for a small trimer fraction). Since both dimers have mass $2 m$, the difference between them cannot be discerned from the analysis of the integrated density profile of the molecules. However the different binding energy and excited dimer 


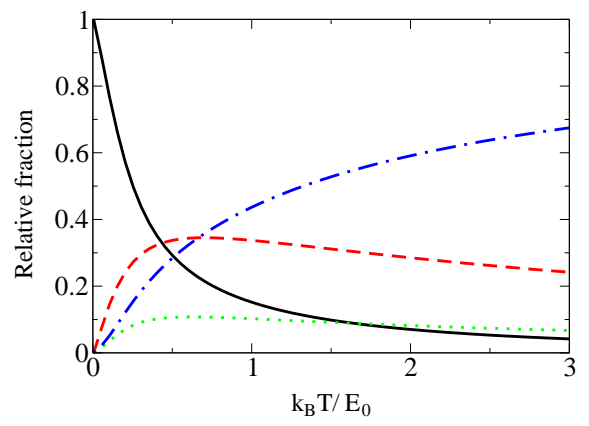

Figure 4: Temperature dependence of the fraction of molecules in trimers (solid), dimers I (dashed), dimers II (dotted) and monomers (dashed-dotted). We consider the parameters of Fig. 3 with $N<N_{c}$.

modes for both types of dimers may be studied spectroscopically to reveal the dual nature of the mixture. If $N \gg N_{c}$ but $U_{0}^{*}(D, I) \ll U_{0}<U_{0}^{*}(D, I I)$, dimers II are precluded, and hence the DCL will become at low $T$ a Bose-Fermi mixture of dimer-I bosons and degenerate monomers at sites 1 and 3 (which act as a pseudospin-1/2 fermions). Again, this exotic mixture could be revealed from the corresponding dual density profile.

The discussed ideal gas analysis allows for a relatively easy understanding of key qualitative features characterizing fermionic polar molecules in deep optical lattices under more general conditions, as the competition between filament-binding energy and Fermi energy of fermionic chains, the relevant role of the filament modes at zero and finite $T$, or the formation of peculiar mixtures of different types of composite bosons and fermions. However, the quantitative validity of the ideal gas approximation is rather limited (also for bosonic molecules [13]), even for the previously discussed 1D arrangement with the particular choice for the dipole orientation. We may estimate the importance of the interfilament interactions by comparing the inter-trimer interactions with the Fermi energy of rigid trimers $\left(\epsilon_{F}=\right.$ $N \hbar \omega)$. For deeply bound chains $\left(R_{0} \sim \Delta / 2\right)$ and at interfilament distances $x>\Delta$ we may approximate the interaction between molecules at different chains as that between two point dipoles. Adding up these interactions we may estimate the mean inter-trimer DDI $V_{f f}(\bar{x})$, where $\bar{x}$ is the mean inter-trimer distance. For the case of $d=0.8$ Debye, $m=100$ atomic mass units, $\Delta=0.5 \mu \mathrm{m}$, and $\omega / 2 \pi=1 \mathrm{~Hz}$, we obtain $U_{0} \simeq 2$, and $N_{c} \simeq 230$. For this value $\bar{x} \simeq 1.7 \Delta$ and $V_{f f} \simeq 0.33 \epsilon_{F}$. The ideal gas approximation is hence quantitatively valid only for dilute mesoscopic samples (as those considered in our numerical calculations). Once the dimer Bose gas nucleates at the trap center the ideal gas condition is quickly violated, due to the larger bosonic densities, although the formation of the dual density profile (similar to that in Fig. 3) still holds. For stacks of 2D sites the ideal gas approximation fails even for extremely low 2D densities. However, the formation of dimer mixtures beyond a given critical density should also occur for 2D arrangements. These mixtures may be considered as weakly-interacting for $2 \mathrm{D}$ densities $n$ such that $n r_{*}^{2}<1$ with $r_{*}=m d^{2} / \hbar^{2}$. For the previous values this demands $n \lesssim 1.1 \times 10^{8} \mathrm{~cm}^{-2}$. For $N \gg N_{c}$ (and $\left.U_{0}>U_{0}^{*}(D, I I)\right)$ weakly-interacting dimers will form a BEC of three different bosons (dimers I in $1-2$, I in 2-3, and II), whose properties will largely depend on the precise determination of the different interdimer interactions, which will be the subject of a future work.

Summarizing, fermionic polar molecules in three-well potentials are expected to form a rather peculiar filament gas. Depending on the filling per site and the interaction strength we expect that the character of the chain gas ranges from a pure trimer gas at low fillings, to a bosonic mixture of pseudo-spin-1/2 and spin-less dimers for large-enough fillings and dipole strengths. Note, finally, that molecules in even larger number of sites may form a quantum gas mixture of increasing complexity. Dipolar chain liquids are hence an exciting perspective for on-going experiments with polar fermionic molecules.

We thank G. Shlyapnikov for useful discussions. This work was supported by the DFG (SFB407, QUEST), and the ESF (EUROQUASAR).

[1] Th. Lahaye et al., arXiv:0905.0386.

[2] M. A. Baranov, Physics Reports 2008 464, 71.

[3] A. Griesmaier et al., Phys. Rev. Lett. 94, 160401 (2005); J. Stuhler et al. Phys. Rev. Lett. 95150406 (2005); Q. Beaufils et al., Phys. Rev. A 77, 061601(R) (2008); Th. Lahaye et al. Nature 448, 672 (2007).

[4] M. Vengalattore et al., Phys. Rev. Lett. 100, 170403 (2008).

[5] M. Fattori et al., Phys. Rev. Lett. 101, 190405 (2008).

[6] S. E. Pollack et al., Phys. Rev. Lett. 102, 090402 (2009).

[7] K. K. Ni et al., Science 322, 231 (2008)

[8] S.Ospelkaus et al., Nature Phys. 4, 622 (2008).

[9] J. Deiglmayr et al., Phys. Rev. Lett. 101, 133004 (2008).

[10] R. Nath, P. Pedri, and L. Santos, Phys. Rev. A 76, 013606 (2007).

[11] M. Klawunn and L. Santos, arXiv:0812.3543

[12] A. Argüelles and L. Santos, Phys. Rev. A 75, 053613 (2007).

[13] D. Wang, M. D. Lukin, E. Demler, Phys. Rev. Lett. 97, 180413 (2006).

[14] P. G. de Gennes and P. A. Pincus, Phys. Kondens.Mater. 11, 189 (1970).

[15] For a review see e.g. P. I. C. Teixeira, J. M. Tavares and M. M. Telo da Gama, J. Phys. Condens.Matter 12, R411 (2000).

[16] K. Butter et al., Nat. Mater. 2, 88 (2003).

[17] M. Klokkenburg et al., Phys. Rev. Lett. 96, 037203 (2006).

[18] For simplicity we neglect that the minimal-energy configuration is slightly tilted from the vertical with an angle $\sim \pi / 10$. 